\title{
MEASUREMENT OF SYNCHROTRON PULSE DURATIONS USING SURFACE PHOTOVOLTAGE TRANSIENTS
}

\author{
T.E. Glover ${ }^{1}$, G.D. Ackermann ${ }^{1}$, A. Belkacem ${ }^{2}$, B. Feinberg ${ }^{1}$, P.A. Heimann ${ }^{1}$, Z. Hussain ${ }^{1}$, \\ H.A. Padmore ${ }^{1}$, C. Ray $^{2}$, R.W. Schoenlein ${ }^{3}$, W.F. Steele ${ }^{1}$. \\ ${ }^{1}$ Advanced Light Source Division, ${ }^{2}$ Chemical Sciences Division, ${ }^{3}$ Materials Sciences Division \\ Lawrence Berkeley National Laboratory \\ 1 Cyclotron Road, MS 2-345 \\ Berkeley, California 94720, USA
}

\begin{abstract}
We report results on experiments using combined laser and synchrotron radiation. Picosecond laser pulses at $800 \mathrm{~nm}$ are used to induce surface photovoltage transients in p-type Si samples. A two-component decay is observed. The fast component of decay provides a direct measure of synchrotron soft $\mathrm{x}$-ray pulse durations.
\end{abstract}

PACS : 42.50.Hz, 32.80.Wr

Keywords : Combined laser-synchrotron spectroscopy; x-ray pulse measurement.

Correspondence author :

T. Ernest Glover MS 2-345, Lawrence Berkeley National Laboratory, 1 Cyclotron Road, Berkeley, CA. 94720, USA.

phone : $510-486-6556$

fax : 510-486-5530

email : teglover@lbl.gov 
Processes driven by combined synchrotron (x-ray) and laser (optical) radiation provide a basis for novel scientific directions and technological applications of synchrotron light. From a fundamental perspective, synchrotron x-rays can probe transient states of matter initiated by laser excitation. From an applications perspective, x-ray interactions with a laser-perturbed material may be used to characterize synchrotron x-ray pulses. The surface photovoltage effect has been studied previously [1,2] and provides an opportunity for such synchrotron x-ray pulse characterization. We describe time-resolved measurements of Si $2 p$ photoemission peak shifts induced by surface photovoltage transients. The photoemission peak dynamics provide a direct measure of the temporal duration of soft x-ray pulses at the Advanced Light Source (ALS).

Experiments are performed at the ALS micro-XPS beamline (BL 7.3.1.2). The beamline provides a micro-focused x-ray spot (diameter $<5 \mu \mathrm{m}$ ) and typically delivers a flux of $\sim 10^{10}$ $\gamma / \sec ^{*} 0.1 \%$ BW. Soft x-ray photons at $\sim 700 \mathrm{eV}$ photoionize a p-type Si (111) sample and the xray photoelectron spectrum (XPS) is measured using a commercial hemispherical analyzer. Perturbations to the XPS are induced by a laser. The laser system is based on titanium-dopedsapphire and provides $800 \mathrm{~nm}$ pulses at a repetition rate of $\sim 83 \mathrm{MHz}(12 \mathrm{~ns}$ laser pulse spacing). The laser pulse energy is typically 1-2 $\mathrm{nJ}$ per pulse and the laser beam is focused to a $15 \mu \mathrm{m}$ diameter spot resulting in an excitation fluence of $\sim 1 \mathrm{~mJ} / \mathrm{cm}^{2}$. Laser pulses are temporally stretched in a fiber to a pulse duration of $5 \mathrm{ps}$. The laser system is synchronized to the ALS storage ring (typically 1-2 ps RMS jitter) by driving a laser-cavity end-mirror with a piezoelectric actuator which in turn is driven by a phase-locked loop (whose inputs are an ALS RF signal and a laser photodiode signal).

The x-ray/laser spatio-temporal overlap is achieved as follows. Coarse spatial overlap is achieved by viewing a phosphor-coated region of the Si sample and overlapping X-ray and laser 
beams on the phosphor. Fine spatial overlap is then obtained by translating the sample to a phosphor-free region and scanning the laser focal spot until a shift is observed in the Si $2 p$ photoemission peak. We note that the Si $2 p$ photovoltage shift does not fully relax between laser excitation pulses so that temporal overlap is not required to observe a Si $2 p$ XPS shift (temporal overlap does of course maximize the shift). Using the above-described procedure and computercontrolled scanning of the laser focal spot (implemented using piezoelectric actuators and linear variable differential transformers), optimal overlap of the two sub-20 $\mu \mathrm{m}$ spots is easily and efficiently achieved.

Coarse temporal overlap is achieved using an avalanche photodiode (pulses overlapped typically to within $500 \mathrm{ps}$ ). The $\mathrm{x}$-ray/laser time-delay is then optimized on a sub-500 ps timescale by scanning the time delay and maximizing the Si $2 p$ peak shift. The $x$-ray/laser time delay is varied by shifting the phase of the ALS RF signal before it is sent into the phaselocked-loop. This method of varying the x-ray/laser time delay is attractive since it is purely electronic and physical motion of (laser) beam steering optics is avoided. Consequently the xray/laser spatial overlap is not compromised. In order to match the laser repetition rate, the Advanced Light Source is operated in a special mode whereupon every $6^{\text {th }}$ bucket is filled (resulting in a 12 ns spacing between ALS pulses).

A laser induced shift to the Si 2 p peak can be observed in Fig. 1 inset (dashed vs solid curves). One observes a laser-induced shift of $\sim 450 \mathrm{meV}$ to lower binding energy. Similar XPS shifts have been observed previously in Si and are due to a surface photovoltage [1]. In brief, the laser produces (photo-generated) carriers and these carriers move to offset band bending at the surface of the p-type Si sample. This carrier motion is rapid (typically sub-picosecond) and is unresolved in the present experiment (where time-resolution is set by the $\sim 70$ ps ALS pulse duration in this operating mode). This rapid carrier motion causes the XPS shift observed in Fig. 
1 (inset). The data in the inset is obtained by recording spectra either with or without a laser pulse so that one either observes no shift or the full surface photovoltage shift. We have observed that the magnitude of the shift varies with surface quality. Smaller shifts (down to $\sim 0$ $\mathrm{meV}$ ) are obtained if the Si surface is damaged from very high laser excitation fluence (in the $\mathrm{J} / \mathrm{cm}^{2}$ regime). Similarly the presence of surface contaminants or oxygen coverage can alter the magnitude of the shift.

Surface photovoltage dynamics are shown in Fig. 1 (main figure). In these measurements a laser always excites the Si and we measure the XPS shift (specifically electron counts over a spectral range from 101-102 eV) as a function of x-ray/laser relative arrival time at the sample. Under these conditions the XPS shift does not fully relax between laser excitation pulses. At short time-delay $(<350 \mathrm{ps})$ the $\mathrm{x}$-ray probes the sample before laser excitation (here the x-ray sees a residual shift from the previous laser pulse). At a delay of $\sim 350$ ps the $\mathrm{x}$-ray and laser pulse start to arrive simultaneously at the Si sample; a larger XPS shift is observed and one observes an increase in the electron count rate. The electron count rate will subsequently decrease as the XPS shift relaxes due to loss of photo-carriers (either from recombination ,transport to the bulk...[1]).

The data of Fig. 1 indicates that there are two components which govern relaxation of the XPS shift. A slow component is observed beyond 500 ps; similar 'slow' dynamics have been observed previously [1] and are attributable to carrier transport within the space-charge layer of the semiconductor. A 'fast' component of decay is observed between 400 and 500 ps and is likely due to rapid electron-hole recombination at the semiconductor surface. Surface recombination is thought to be mediated by trap states (which lie within the semiconductor bandgap) and as such will depend on the nature of the surface[1-3]. Measurements of surface dynamics on annealed $\operatorname{Si}(111) 2 \times 1$ surfaces indicate surface carrier decay times of order 100 ps 
$[2,3]$. The samples used in the present experiments are Ar-ion sputtered $\operatorname{Si}(111) 2 \times 1$ samples and the measurements of Fig. 1 indicate similar, though somewhat faster, carrier decay times. Somewhat faster decay times (compared to annealed surfaces) can result from increased density of trap/defect states associated with ion-sputtering.

The rapid material response associated with surface carrier dynamics gets convolved with the ALS pulse duration and as such provides a measure of the synchrotron pulse duration. We determine the width (full width at half-maximum) of this feature to be $70 \mathrm{ps}$.

In conclusion, we have used combined laser and synchrotron radiation to study the dynamics of surface photovoltage transients in Si. A fast component of photo-carrier decay permits a measure of the temporal duration of synchrotron x-ray pulses.

\section{References}

[1] J.P. Long et al., Phys. Rev. Lett. 64, 1158 (1990).

[2] M. Marsi et al., Appl. Phys. Lett. 70, 895 (1997).

[3] N.J. Halas and J. Bokor, Phys. Rev. Lett. 62, 1679 (1989). 


\section{Figure Caption}

Figure 1. Electron counts (over a 101-102 eV spectral range) from Si $2 p$ photoemission peak vs $\mathrm{x}$-ray/laser time-delay. At early delay $(<350 \mathrm{ps})$ the $\mathrm{x}$-rays probe before laser excitation of the sample. When x-ray and laser pulses strike the Si sample simultaneously (starting at $\sim 350 \mathrm{ps)}$ an increased count rate is obtained due to a shift in the photoelectron peak. Partial relaxation of the shifted peak (occurring from $\sim 400-500$ ps) provides a direct measure of the ALS pulse duration (FWHM $\sim 70 \mathrm{ps})$. The full Si $2 \mathrm{p}$ shift $(\sim 450 \mathrm{meV})$ is shown in the figure inset (dashed curve : no laser; solid curve $=$ laser present). 


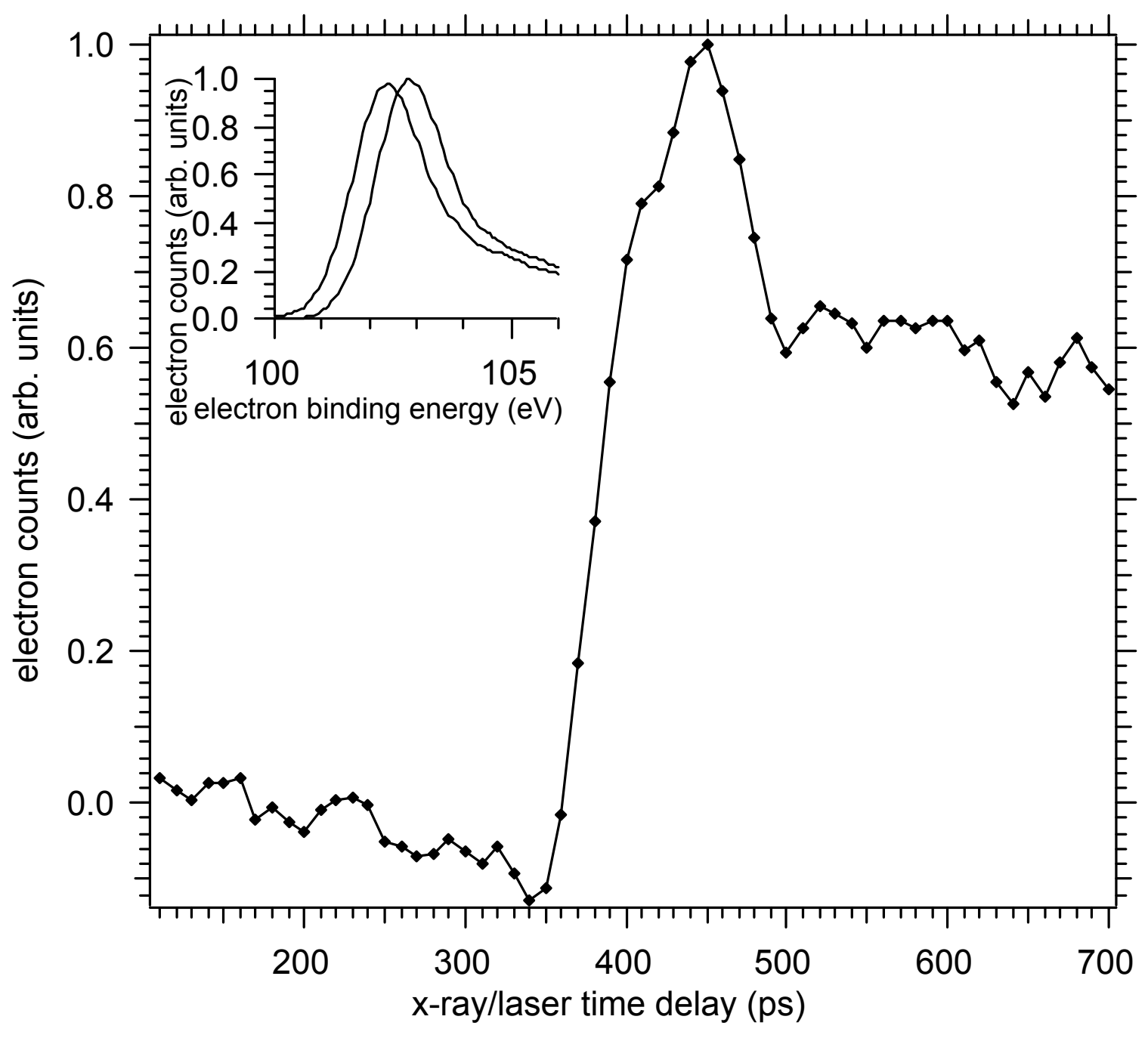

Figure 1 